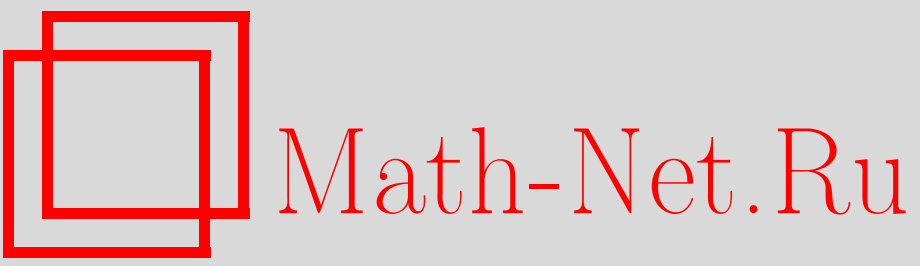

Я. Б. Воробец, Плоские структуры и бильярды в рациональных многоугольниках, УМН, 1996, том 51, выпуск 1, 145-146

DOI: https://doi.org/10.4213/rm925

Использование Общероссийского математического портала Math-Net.Ru подразумевает, что вы прочитали и согласны с пользовательским соглашением

http://www.mathnet.ru/rus/agreement

Параметры загрузки:

IP: 54.166 .219 .16

26 апреля 2023 г., 09:26:20 


\title{
ПЛОСКИЕ СТРУКТУРЫ И БИЛЬЯРДЫ В РАЦИОНАЛЬНЫХ МНОГОУГОЛЬНИКАХ
}

\author{
Я. Б. ВОРОБЕЦ
}

ОПРЕДЕлЕниЕ 1. Плоская структура на компактной и связной ориентируемой поверхности $M$ - это атлас $\omega$, состоящий из карт вида $(U, f)$, где $U$ - область $M, f$ - гомеоморфизм $U$ на область в $\mathbb{R}^{2}$. При этом должны выполняться следующие условия: (i) области $U$ покрывают всю поверхность $M$ кроме конечного числа точек $x_{1}, \ldots, x_{k}$, называемых особыми; (ii) все функции замены координат являются сдвигами в $\mathbb{R}^{2}$; (iii) атлас $\omega$ максимален по отношению к условиям (i), (ii); (iv) для каждой особой точки $x_{i}$ существует проколотая окрестность $\dot{U}_{i}$ и отображение $f_{i}$ этой окрестности на проколотую окрестность $\dot{V}$ точки в $\mathbb{R}^{2}$, являющееся сдвигом в локальных координатах из атласа $\omega$, и такое, что у каждой точки из $\dot{V}$ есть ровно $m_{i}$ прообразов.

Эквивалентно, $\omega$ - это риманова метрика нулевой кривизны на $M \backslash\left\{x_{1}, \ldots, x_{k}\right\}$, имеющая в точке $x_{i}$ коническую особенность с углом $2 \pi m_{i}$. Плоские структуры возникают, например, при рассмотрении бильярдов в рациональных многоугольниках. Именно, конструкция Землякова и Катка [1] сводит такой бильрд к геодезическому потоку на некоторой поверхности с плоской структурой.

В настоящей работе рассматриваются плоские структуры, геодезический поток для которых повторяет свойства геодезического потока на торе $\mathbb{R}^{2} / \mathbb{Z}^{2}$, где каждая траектория либо периодична, либо равнораспределена. Важнейшим результатом здесь является приводимая ниже теорема 1.

ОПРЕДЕЛЕНИЕ 2. Плоская структура $\omega_{1}$ на поверхности $M_{1}$ называется изоморфной $\omega=\{(U, f)\}$, если $\omega_{1}=\left\{\left(f_{1}^{-1}(U), f \circ f_{1}\right)\right\}$ для некоторого гомеоморфизма $f_{1}: M_{1} \rightarrow M$. Стабилизатором $\Gamma(\omega)$ плоской структуры $\omega$ назьвается группа тех элементов $a \in \operatorname{SL}(2, \mathbb{R})$, для которых плоская структура $a \omega=\{(U, a \circ f)\}$ изоморфнна $\omega$.

Седлосвязка плоской структуры $\omega$ - это геодезический отрезок, соединяющий две особые точки и не содержащий особых точек внутри себя. Длину кратчайшей седлосвязки обозначим через $m(\omega)$.

Теорема 1 (альтернатива Вича). Пусть стабилизатор $\Gamma(\omega)$ - решетка в $\mathrm{SL}(2, \mathbb{R})$. Тогда поток на поверхности в произвольном направлении $\bar{v}$ либо имеет только периодические компоненты, либо строго әргодичен. Первая возможнность реализуется в том

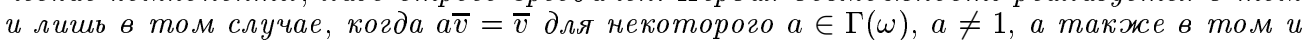
лишь в том случае, когда найдется седлосвязка, параллельная $\bar{v}$.

А. М. Стёпин предложил автору найти доказательство теоремы 1 , в отличие от первоначального (см. [2]) не использующее понятий теории Тейхмюллера. Для этого понадобилось дать новое доказательство следующего утверждения (его формулировка отлична от оригинальной, но эквивалентна ей).

Лемма 1 (Мазур [3]). Пусть плоская структура $\omega$ такова, что $m\left(g^{t} \omega\right) \nrightarrow 0$ при $t \rightarrow+\infty$, где $g^{t}=\left(\begin{array}{cc}e^{t / 2} & 0 \\ 0 & e^{-t / 2}\end{array}\right)$. Тогда поток в вертикальном направлении строго эргодичен.

Поток в вертикальном направлении можно представить в виде специального потока над перекладыванием $T$, где концы перекладываемых интервалов соответствуют особым траекториям. Поток и перекладывание одновременно строго эргодичны или нет. Обозначим через $\varepsilon_{n}(T)$ наименьшую из длин отрезков, перекладываемых перекладыванием $T^{n}$.

ТеОРема 2 (Вич [4]). Если $n \varepsilon_{n}(T) \nrightarrow 0$ при $n \rightarrow \infty$ и перекладывание $T$ минимально, то оно строго эргодично.

Лемма 1 теперь вытекает из следующего утверждения.

Работа выполнена при частичной финансовой поддержке Международного научного фонда (грант № M1E000). 
Лемма 2. Если плоская структура $\omega$ удовлетворяет условиям леммы 1, то перекладывание Т удовлетворяет условиям теоремы 2.

Следуюшие далее теоремы 3 и 4 обобщают и дополняют теорему 1.

ОПРедЕЛЕниЕ 3 . Пусть $\omega$-плоская структура на поверхности $M . \omega-$ треугольником называется треугольник на $M$, у которого вершины - особые точки, стороны - седлосвязки, и внутри которого особых точек нет.

Плоская структура $\omega$ обладает свойством А, если площадь каждого $\omega$-треугольника больше некоторой положительной постоянной. $\omega$ обладает свойством В, если, более того, эта площадь принимает конечное число значений.

ТЕорема 3. Плоская структура, обладающая свойством А, удовлетворяет альтернативе Вича.

Теорема 4. Плоская структура обладает свойством В в том и лишь в том случае, когда ее стабилизатор - решетка.

При доказательстве теоремы 4 используется следующий критерий.

Лемма 3. Дискретная подгруппа $\Gamma \subset \mathrm{SL}(2, \mathbb{R})$ является решеткой в том и лишь в том случае, когда можно выбрать конечное число ненулевых векторов $\bar{v}_{1}, \ldots, \bar{v}_{k}$ таким образом, что множество $S=\Gamma \bar{v}_{1} \cup \cdots \cup \Gamma \bar{v}_{k}$ дискретно и для любого а $\in \operatorname{SL}(2, \mathbb{R})$ найдется вектор $\bar{v} \in S$ такой, что $|a \bar{v}| \leqslant C$, где $C$ - не зависящая от а постоянная.

Примеры плоских структур, удовлетворяюших альтернативе Вича, возникают при рассмотрении бильярда в треугольниках с углами $\frac{\pi}{n}, \frac{\pi}{m}$ и $\pi-\frac{\pi}{n}-\frac{\pi}{m}$, где $n, m$-целые. Соответствующую плоскую структуру обозначим через $\omega_{n, m}$.

ТеОрема 5. Плоские структуры $\omega_{2, n}, \omega_{n, n} \quad(n \geqslant 3), \omega_{n, 2 n} \quad(n \geqslant 2), \omega_{3,4}, \omega_{3,5}$ имеют стабилизатор-решетку.

Исходя из этих примеров (первые два из них приведены в [2]), можно строить более сложные, пользуясь конструкцией накрытия. Накрытием плоских структур $\omega_{1}$ и $\omega_{2}$, заданных на поверхностях $M_{1}$ и $M_{2}$, называется отображение $f: M_{1} \rightarrow M_{2}$, которое переводит особые точки в особые и является сдвигом в локальных координатах из $\omega_{1}$ и $\omega_{2}$.

ТЕОРема 6. Пусть плоская структура $\omega_{1}$ накрывает плоскую структуру $\omega_{2}$. Тогда $\Gamma\left(\omega_{1}\right) \cap \Gamma\left(\omega_{2}\right)$ - подгруппа конечного индекса в каждой из групп $\Gamma\left(\omega_{1}\right)$ и $\Gamma\left(\omega_{2}\right)$. В частности, $\Gamma\left(\omega_{1}\right)$ и $\Gamma\left(\omega_{2}\right)$ одновременно являются или не являются решетками.

В качестве следствия отсюда получаем, что стабилизатор-решетку имеют плоские структуры, соответствующие правильным многоугольникам.

Плоские структуры со стабилизатором-решеткой допускают полное описание периодических траекторий. Это описание позволяет доказать, например, следующую теорему, обобщающую ряд результатов из [5]. Напомним, что периодическая бильярдная траектория в многоугольнике $Q$ назьвается устойчивой, если многоугольник, полученный из $Q$ малым шевелением сторон, содержит периодическую бильярдную траекторию, близкую к исходной.

ТеОРема 7. В прямоугольном треугольнике с острым углом $\pi /(2 n) \quad(n \geqslant 2)$ все периодические бильярдные траектории неустойчивы.

\section{СПИСОК ЛИТЕРАТУРЫ}

[1] Земляков А.Н., Каток А. Б. // Матем. заметки. 1975. Т. 18. №2. С. 291-300. [2] Veech W. A. // Invent. Math. 1989. V. 97. № 4. P. 553-583. [3] Masur H. // Duke Math. J. 1992. V. 66. № 3. P. 387-442. [4] Veech W. A. // Ergod. Th. \& Dynam. Sys. 1987. V. 7. № 1. Р. 149-153. [5] Воробец Я. Б., Гальперин Г. А., Стёпин А. М. // УМН. 1992. Т. 48. № 3. C. $9-74$. 